Document downloaded from:

http://hdl.handle.net/10251/60829

This paper must be cited as:

Jiménez Molero, MC.; Miranda Alonso, MÁ. (2014). Triplet Excited States as a Source of Relevant (Bio)Chemical Information. Current Topics in Medicinal Chemistry. 14(23):27342742. doi:10.2174/1568026614666141216100907.

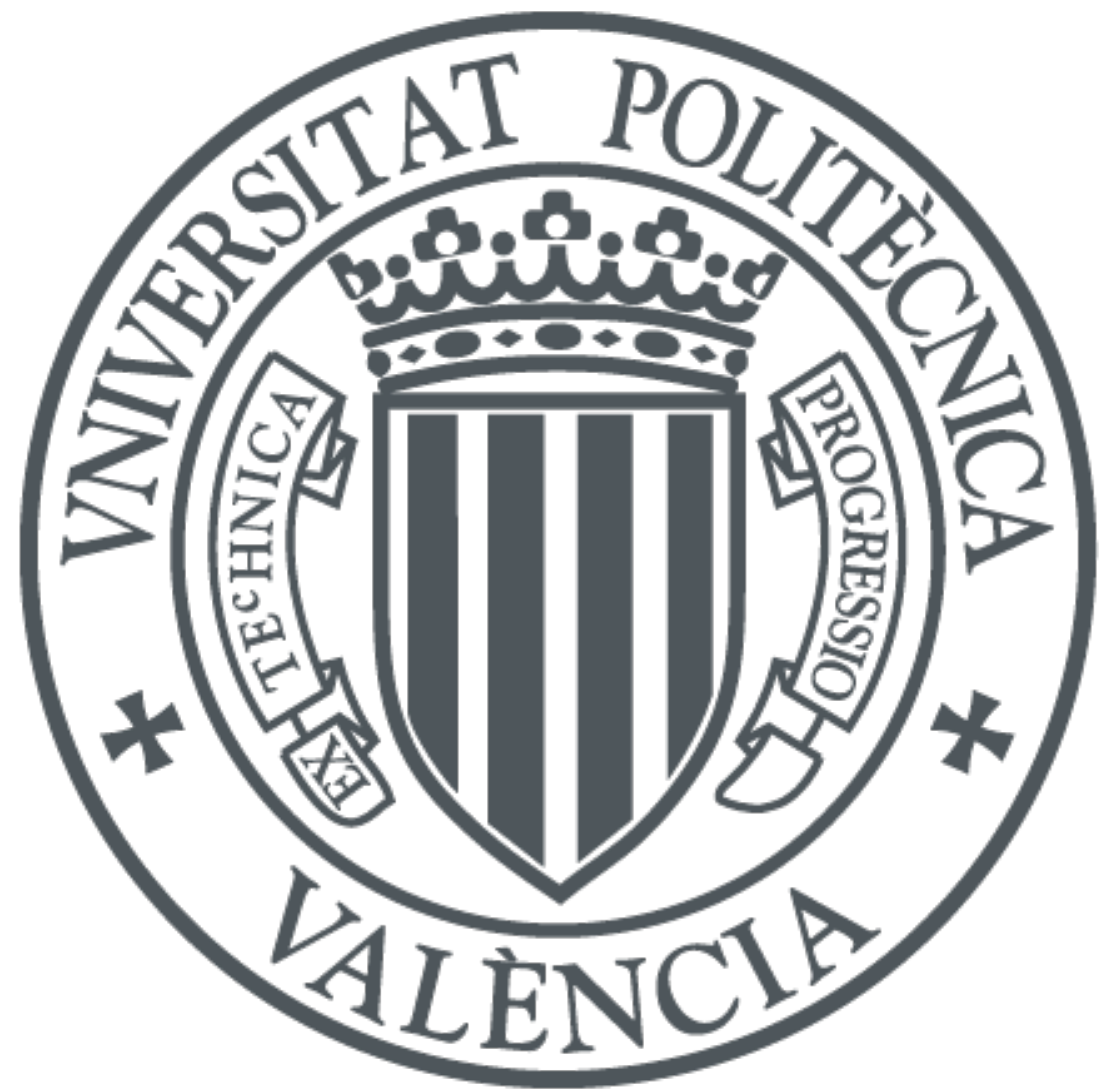

The final publication is available at

http://www.ingentaconnect.com/content/ben/ctmc/2014/00000014/00000023/art00013

Copyright Bentham Science Publishers

Additional Information 


\title{
Triplet Excited States as a Source of Relevant (Bio)Chemical
}

\section{Information}

\author{
M. Consuelo Jiménez* and Miguel A. Miranda \\ Departamento de Química/Instituto de Tecnología Química UPV-CSIC. Universitat \\ Politècnica de València, Camino de Vera s/n, Apdo 22012, E-46071 Valencia (Spain). \\ mcjimene@qim.upv.es; mmiranda@qim.upv.es
}

\begin{abstract}
Triplet excited states exhibit strongly medium-dependent properties, which turns them into valuable tools to probe the microenvironments existing in protein binding pockets. Monitoring of the triplet excited state behavior of drugs within transport proteins (serum albumins and $\alpha_{1}$-acid glycoproteins) by laser flash photolysis constitutes a valuable source of information on interaction strength, conformational restrictions and protection from attack by oxygen or other external reagents. With proteins, formation of encapsulated triplet excited states is favored over other possible processes leading to ionic species. Remarkably, under aerobic conditions, the triplet lifetimes of drug@protein complexes are dramatically longer than in bulk solution. This offers a broad dynamic range for identification of distinct triplet populations or for chiral discrimination. In this review, specific applications of the laser flash photolysis technique are described, including determination of drug distribution among the bulk solution and the protein binding sites, competition of two types of proteins to bind a drug, occurrence of drugdrug interactions within protein binding sites, and enzymatic-like activity of the proteins. Finally, protein encapsulation can also modify the photoreactivity of the guest. This is illustrated by presenting an example of retarded photooxidation.
\end{abstract}




\section{Graphical abstract}

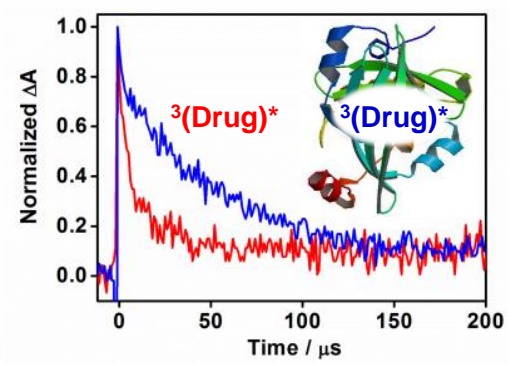

Keywords: $\alpha_{1}$-acid glycoproteins, drug, laser flash photolysis, serum albumins, protein binding, transient absorption spectroscopy. 


\section{Introduction}

In the laser flash photolysis (LFP) technique, a short pulse of light (nano or femtoseconds) from a laser is used to interact with a sample that is located in the optical path of a spectrometer; the result of this interaction can be either a transient absorption or emission process. The signal detected following laser excitation of a substrate may be due to a variety of transient species such as i) triplet excited states (TES) formed after electronic excitation followed by intersystem crossing, ii) radicals obtained by homolytic bond cleavage, iii) radical ions resulting from electron transfer, iv) isomers obtained after rearrangement, etc. ${ }^{1}$ (Scheme 1$)$.

$$
\begin{aligned}
& \mathrm{A} \longrightarrow{ }^{\mathrm{hv}} \longrightarrow{ }^{1} \mathrm{~A}^{*} \\
& { }^{1} \mathrm{~A}^{*} \stackrel{\mathrm{ISC}}{\longrightarrow}{ }^{3} \mathrm{~A}^{*} \\
& { }^{1} \mathrm{~A}^{*} \text { or }{ }^{3} \mathrm{~A}^{*} \longrightarrow \mathrm{a}^{\bullet}+\mathrm{b}^{\bullet} \\
& { }^{1} \mathrm{~A}^{*} \text { or }{ }^{3} \mathrm{~A}^{*} \longrightarrow \mathrm{A}^{+.} \text {or } \mathrm{A}^{-} \\
& { }^{1} \mathrm{~A}^{*} \text { or }{ }^{3} \mathrm{~A}^{*} \longrightarrow \mathrm{A}^{\prime}
\end{aligned}
$$

Scheme 1. Transient species detectable by LFP.

Transient absorption spectroscopy (TAS) has been much less exploited than fluorescence emission to investigate bio(chemical) issues, although it constitutes a powerful tool for this purpose. A schematic representation of an LFP equipment are shown in Figure 1. 


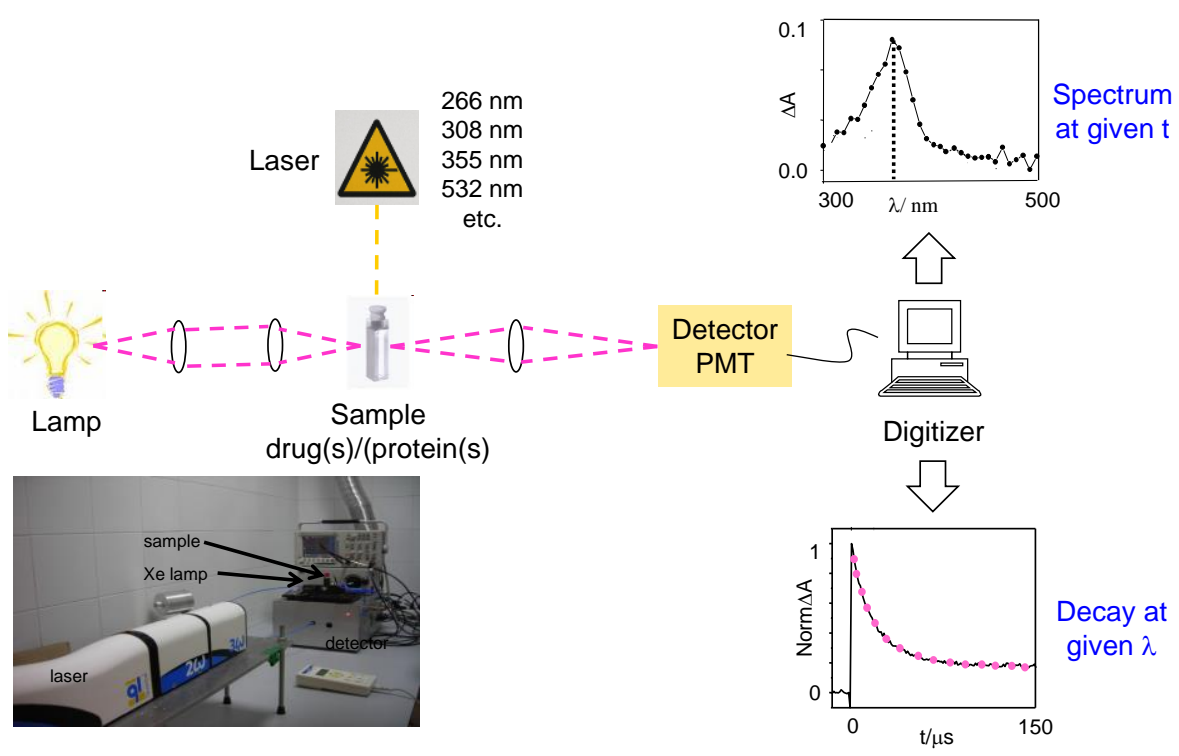

Figure 1. Schematic representation of an LFP setup.

The behavior of TES of ligands after protein encapsulation can be easily monitored by employing LFP. The properties of TES (especially the triplet lifetimes, $\tau_{\mathrm{T}}$ ) are very sensitive to the microenvironment experienced within the protein binding sites, and thus they can be successfully employed to investigate the issue of drug binding (see cartoon in Figure 2), providing information on interaction strength, conformational restrictions, protection from attack by oxygen or other reagents, stereochemical requirements, etc.

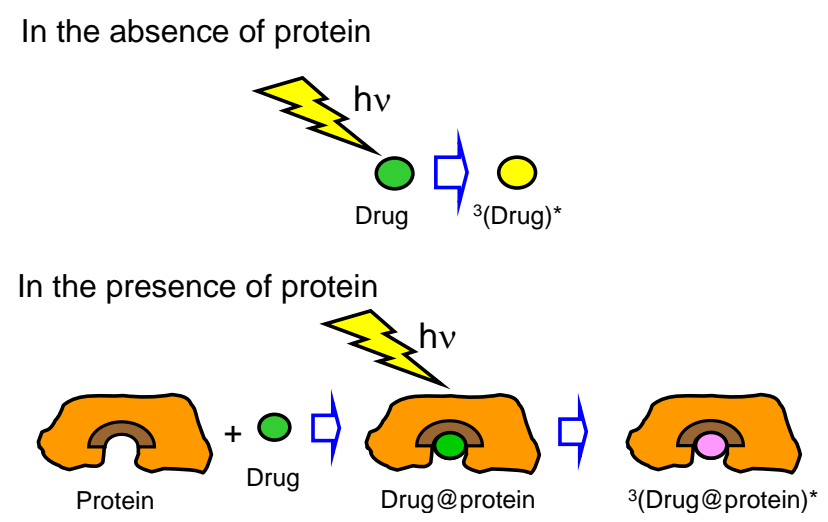

Figure 2. Principle of the LFP-based approach described in this review. 
Drugs suitable for monitoring TES within proteins should possess a chromophore able to absorb the laser irradiation, with a non-negligible intersystem crossing quantum yield and a triplet lifetime in the nanosecond-microsecond time scale. For this purpose, drugs containing chromophores such as biphenyl (flurbiprofen), naphthalene (naproxen, cinacalcet, propranolol, nabumetone) or anthracene (anthracenealkanoic acids) are specially appropriate. As additional requirements, the protein should not absorb or it should not produce any signal in the wavelength region where the drug intermediates do, and the product of the molar absorption coefficient of the excited state by the intersystem crossing quantum yield has to remain constant in the absence and presence of protein.

Most of the work on the use of TES for obtaining relevant bio(chemical) information has been done with transport proteins (TP), such as human and bovine serum albumin (HSA, BSA) or $\alpha_{1}$-acid glycoproteins (HAAG, BAAG). These proteins are very abundant in plasma and are responsible for a variety of relevant processes, although one of their most important physiological roles is to carry different agents (i.e. drugs or metabolites) in the bloodstream for their selective delivery to specific targets. ${ }^{2,3,4}$ In addition, the role of plasma protein binding has been recognized as an important factor in drug disposition and efficacy. ${ }^{5}$ In this context, drug binding to TP is a key process, which is involved in phenomena such as modulation of drugs solubility in plasma, toxicity, in vivo half-life, etc. ${ }^{6}$ Concerning serum albumins, they are major carriers of small organic molecules, which bind primarily to two high-affinity sites (called site I or warfarin binding site and site II or indole-benzodiazepine binding site), with typical association constants in the range of $10^{4}-10^{6} \mathrm{M}^{-1} .^{7}$ As regards $\alpha_{1}$-acid glycoproteins, their concentration in serum is much lower than that of albumins. For HAAG, up to seven binding sites have been described, although most small organic molecules bind exclusively to one of them. ${ }^{8,9}$ This high-affinity site is large and flexible, and the areas for basic, acid, and neutral drugs 
overlap. For BAAG, a substantial overlap of a basic drug and a steroid hormone binding sites has been described.

Binding of drugs to TP has been studied for years with different methodologies, in order to understand their functions and to disclose the structural basis for designing new therapeutic agents. The techniques include equilibrium dialysis, high performance liquid chromatography, ultrafiltration, spectrophotometry, calorimetry, circular dichroism, capillary electrophoresis, nuclear magnetic resonance, etc. ${ }^{10}$ Although in general these techniques are useful and widely employed, their main disadvantages can be related to sensitivity, interferences, diffusion problems or lack of reproducibility arising from a complicated workup. Some of these problems can be circumvented by means of TAS, which can be envisaged as a complementary tool. The LFP-based approach is fast and simple, as it may require just one measurement per sample to provide accurate results. It is highly sensitive and is appropriate for analysis of minute $(\mu \mathrm{g})$ amounts of sample. As further advantages, it avoids work-up, substrate derivatization and chromatographic separation. $^{11}$

In this review, some representative applications of the LFP technique on drug/transport protein systems are detailed. They include issues such as the determination of drug distribution among the bulk solution and the protein binding sites, competition of two proteins to bind a ligand, drug-drug interactions within protein binding sites, enzymaticlike activity of serum albumin or determination of enantiomeric compositions. The chemical structures of the drugs or ligands whose behavior is described in this review are shown in Chart 1. 


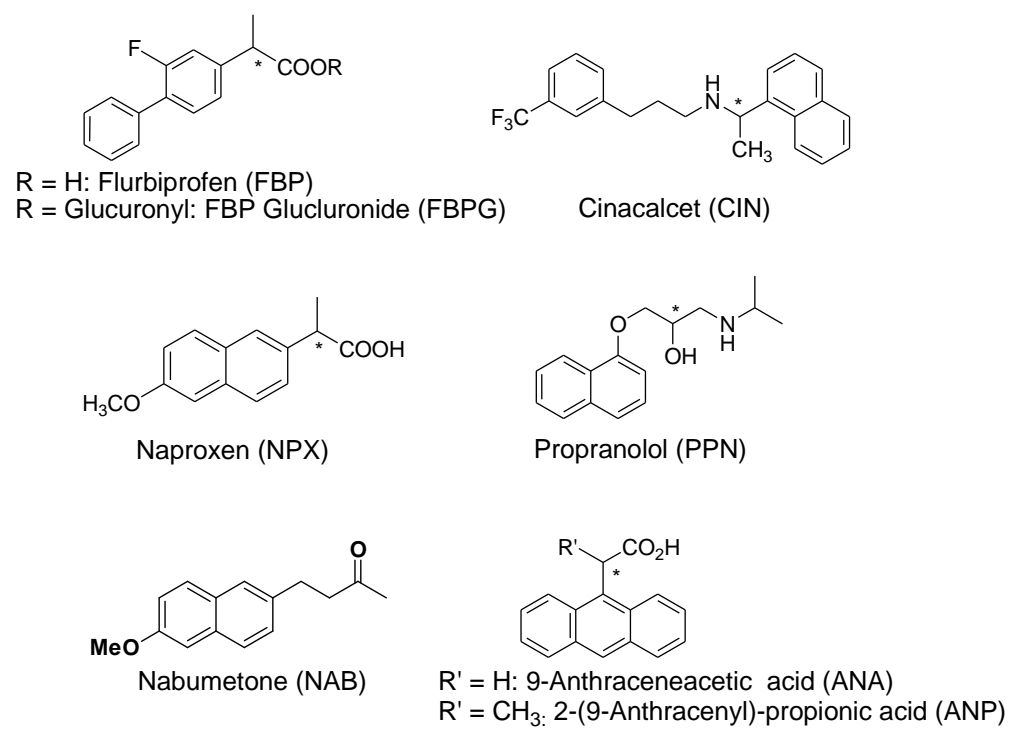

Chart 1. Chemical structures of the drugs and ligands described in this work.

\section{Drug binding within the protein binding sites}

When drugs are bound to proteins, their time of action is prolonged by retarding metabolization. Only unbound drugs are pharmacologically active. The extent of binding depends on total drug concentrations and on protein affinity. The process is usually reversible, and equilibrium is established between bound and free drug. A high protein binding affinity results in improved solubility and increased half-life. Conversely, a low value of this parameter is associated with limited ability to perfuse tissues and to reach the site of action. ${ }^{12,13,14}$

In this context, LFP-based methodologies have been developed to assess protein occupancy in the presence of one or two different transport proteins and to reveal drugdrug interactions between two drugs that share a common protein binding site. 


\subsection{Assesment of protein occupancy}

A well-documented example on the use of TAS to establish protein occupancy degree is the case of the non-steroidal anti-inflammatory drug flurbiprofen ${ }^{15}$ (FBP, Chart 1), which belongs to the arylpropionic acid family and contains a chiral center. The transient spectrum of (S)-FBP obtained after laser excitation at $266 \mathrm{~nm}$ (PBS, air), displays a maximum at $360 \mathrm{~nm}$ (Figure $3 \mathrm{~A})$, assigned to the FBP triplet excited state $\left({ }^{3} \mathrm{FBP} *\right) .{ }^{16}$
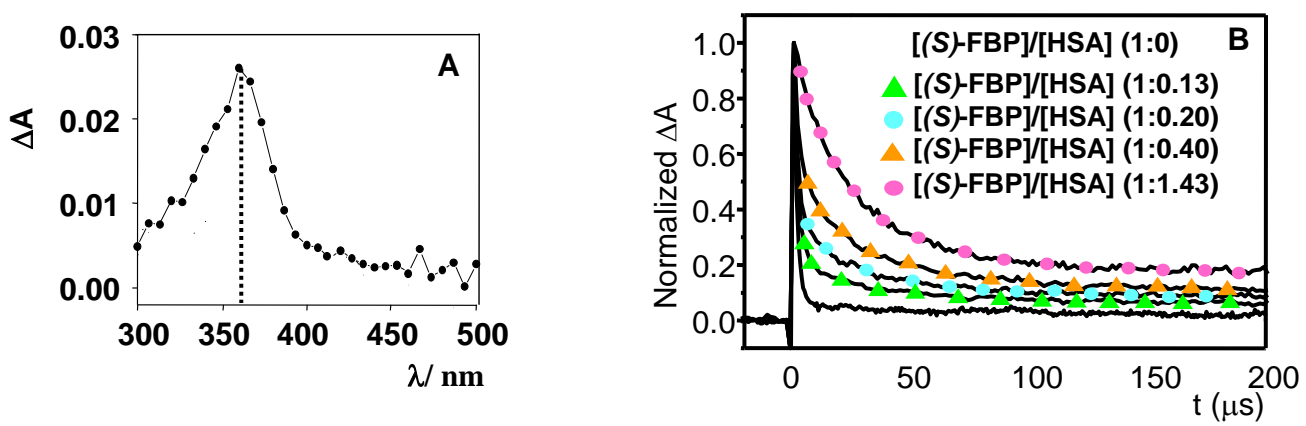

Figure 3. Laser flash photolysis $\left(\lambda_{\text {exc }}=266 \mathrm{~nm}\right)$ of $(S)$-FBP $\left(2.5 \times 10^{-5}\right.$, PBS, air $)$. A: Transient spectrum obtained $0.5 \mu$ s after the laser pulse. B: Kinetic decays monitored at $\lambda=360 \mathrm{~nm}$ after addition of different amounts of HSA.

The kinetic decay trace (Figure 3B) follows a first order exponential law with a lifetime $\left(\tau_{\mathrm{F}}\right)$ of $1.5 \mu$ s (Eq. 1).

$$
\Delta \mathrm{A}=\Delta \mathrm{A}_{0}+\mathrm{A}_{\mathrm{F}} \times \mathrm{e}^{\left(-\mathrm{t} / \tau_{\mathrm{F}}\right)} \quad \text { (Eq. 1) }
$$

After addition of HSA (0.7:1 drug/protein molar ratio), ${ }^{3} \mathrm{FBP} *$ is still the only transient species detected, but the kinetic trace at $\lambda_{\max }=360 \mathrm{~nm}$ is now longer lived (Figure 3B); two monoexponential terms are now necessary for fitting (Eq. 2), with lifetimes of 11.2 and $35.9 \mu$ s (minor and major components, respectively).

$$
\Delta \mathrm{A}=\Delta \mathrm{A}_{0}+\mathrm{A}_{\mathrm{I}} \times \mathrm{e}^{\left(-\mathrm{t} / \tau_{\mathrm{I}}\right)}+\mathrm{A}_{\mathrm{II}} \times \mathrm{e}^{\left(-\mathrm{t} / \tau_{\mathrm{II}}\right)} \quad \text { (Eq. 2) }
$$

They are ascribed to FBP within site I $\left(\tau_{\mathrm{I}}\right)$ and site II ( $\left.\tau_{\mathrm{II}}\right)$ of HSA, respectively, based on the known higher affinity of FBP for site II. $^{17}$ To obtain independent evidence 
supporting this assignment, LFP experiments have been performed in the presence of $(S)$ ibuprofen $\left((S)\right.$-IBP) as site II-probe. ${ }^{18}$ Addition of $(S)$-IBP to the $(S)$-FBP/HSA mixtures results in a decreased contribution of the longest lifetime component, concomitant to an enhancement of the components assigned to free and site-I-bound (S)-FBP (Figure 4).

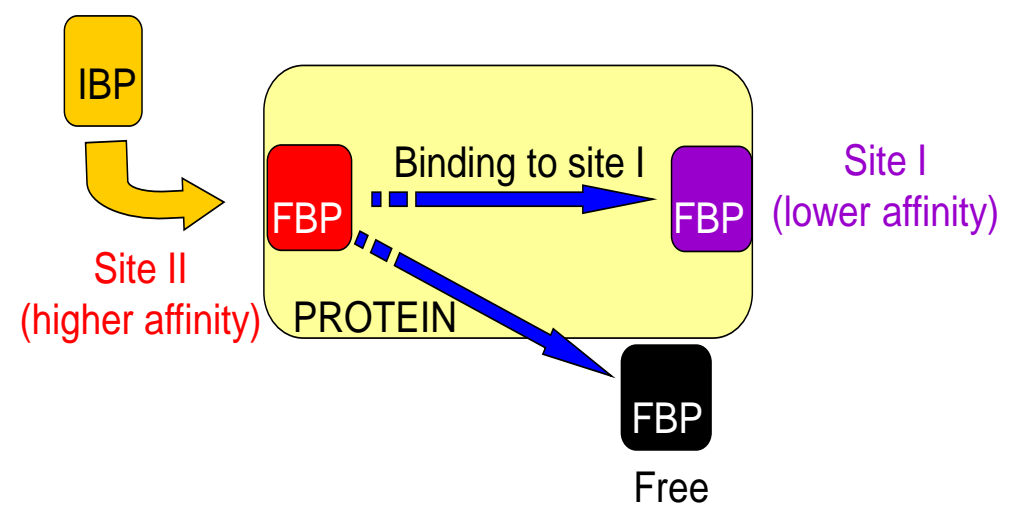

Figure 4. Displacement experiments in the presence of IBP.

For solutions prepared at different drug/protein molar ratios (from 1.4:1 to 0.3:1), fitting of the decay traces contains three monoexponential terms (Eq. 3), supporting distribution of the drug among the two protein binding sites and the bulk solution.

$$
\Delta \mathrm{A}=\Delta \mathrm{A}_{0}+\mathrm{A}_{\mathrm{F}} \times \mathrm{e}^{\left(-\mathrm{t} / \tau_{\mathrm{F}}\right)}+\mathrm{A}_{\mathrm{I}} \times \mathrm{e}^{\left(-\mathrm{t} / \tau_{\mathrm{I}}\right)}+\mathrm{A}_{\mathrm{II}} \times \mathrm{e}^{\left(-\mathrm{t} / \tau_{\mathrm{II}}\right)}
$$

In this equation, subscripts F, I and II indicate contribution of free, site I-bound and site II-bound drug, respectively. After dividing each preexponential coefficient $A_{F}, A_{I}$ or $A_{I I}$ by the sum of the three $A_{i}$ values $\left(A_{F}+A_{I}+A_{I I}\right)$, the percentage of free and bound drug at different amounts of HSA is determined (Figure 5). It can be shown that site II is selectively populated at low $(S)$-FBP/HSA molar ratios, whereas at high ratios most of the drug remains unbound. 


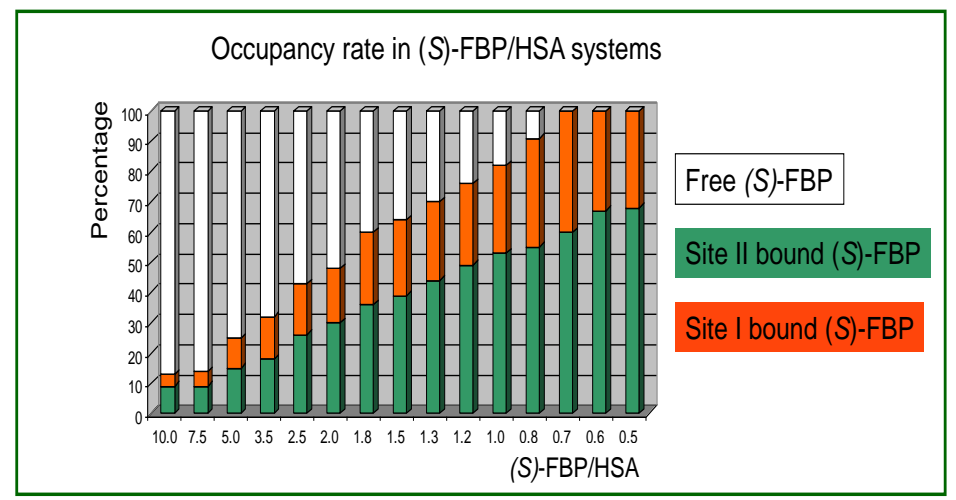

Figure 5. $(S)$-FBP distribution in HSA at different drug/protein molar ratios.

The work has been extended to $(R)$-FBP in HSA and to both enantiomers in BSA. The most significant differences between both albumins are related to the dynamic ranges found for the triplet lifetimes and to the relative occupancy of the two binding sites.

In general, the lengthening of the $\tau_{\mathrm{T}}$ values when encapsulated within proteins indicates a lower reactivity of the TES, which is mainly attributed to protection of ${ }^{3} \mathrm{FBP} *$ from attack by oxygen, which is supported by the high value of the rate constant for triplet quenching by oxygen for free $(S)$-FBP in PBS $\left(1 \times 10^{10} \mathrm{M}^{-1} \mathrm{~s}^{-1}\right)$, compared to the values in site I or site II, that are one and two orders of magnitude lower, respectively. In addition, triplet decay by nonradiative processes is also retarded, due to a limitation of the degrees of freedom inside the TP cavities. The methodology has also been successfully applied to establish the protein binding behavior of FBP methyl esther (a prodrug). ${ }^{19}$

\subsection{Determination of drug distribution in a binary protein system}

A new methodology aimed at a rapid and reliable assessment of drug distribution between two proteins present simultaneously in a biological medium has been developed, based on the determination of the relative contributions of the triplet lifetime components 
obtained from the decay curves in the presence of serum albumin/glycoprotein binary systems. ${ }^{20}$ A representative example is provided by the study of three naphthalenederived drugs,${ }^{21}$ with a (known) different affinity for the two types of proteins: cinacalcet $\left(\mathrm{CIN}\right.$, a calcimimetic), ${ }^{22}$ naproxen (NPX, a NSAID) ${ }^{23}$ and propranolol (PPN, an antihypertensive). ${ }^{24}$ Their chemical structures can be found in Chart 1.

Prior to analysis of the binary systems, the binding behavior in the presence of isolated proteins is established. Starting with CIN, the excited states of the $(R)$-enantiomer have been characterized in order to obtain relevant information about its complexation with HSA. $^{25}$ Upon LFP of $(R)$-CIN@HSA $\left(\lambda_{\text {exc }}=308 \mathrm{~nm}\right.$, air $)$, naphthalene-like TES are generated, with absorption maxima at $420 \mathrm{~nm}$. The corresponding decays are satisfactorily fitted using a function containing two monoexponential terms, corresponding to a short-lived and a long-lived component ( $8 \mu \mathrm{s}$ and $37 \mu \mathrm{s}$, respectively), different from the value found in pure PBS $(1.9 \mu$ s), indicating that the drug is incorporated into two different binding sites of HSA. Interestingly $(R)$-CIN exhibits also a significant affinity towards glycoproteins, although the drug is located within the only existing binding site (Eq. 4, with subscripts $\mathrm{F}$ and $\mathrm{B}$ indicating contribution of free and HAAG bound drug, respectively), with a triplet lifetime of $15 \mu$ s in HAAG.

$$
\Delta \mathrm{A}=\Delta \mathrm{A}_{0}+\mathrm{A}_{\mathrm{F}} \times \mathrm{e}^{\left(-\mathrm{t} / \tau_{\mathrm{F}}\right)}+\mathrm{A}_{\mathrm{B}} \times \mathrm{e}^{\left(-\mathrm{t} / \tau_{\mathrm{B}}\right)}
$$

Likewise, upon LFP of $(S)$-PPN at $\lambda_{\mathrm{exc}}=308 \mathrm{~nm}$, the transient spectrum shows contributions due to ${ }^{3} \mathrm{PPN}^{*}$ and the radical cation $\left(\mathrm{PPN}^{+}\right)$. From the decay traces monitored at $420 \mathrm{~nm}$ under aerobic conditions, their lifetimes have been determined as 1.9 and $13.5 \mu$ s, respectively. The spectra in the presence and in the absence of albumins are almost coincident, which is in good agreement with negligible PPN binding to these proteins. However, in the presence of glycoproteins, the kinetics are markedly different: the decay traces lengthen and require a function containing three monoexponential terms 
(Eq. 5, with superscripts $\mathrm{T}$ and $\mathrm{RC}$ indicating contribution of triplet and radical cation species, respectively) for a satisfactory fitting, corresponding to two free species $\left({ }^{3} \mathrm{PPN} *\right.$ and $\mathrm{PPN}^{+}$) plus the longer lived protein-bound triplet.

$$
\Delta \mathrm{A}=\Delta \mathrm{A}_{0}+\mathrm{A}_{\mathrm{F}}^{\mathrm{T}} \times \mathrm{e}^{\left(-\mathrm{t} / \tau_{F}^{T}\right)}+\mathrm{A}_{\mathrm{B}}^{\mathrm{T}} \times \mathrm{e}^{\left(-\mathrm{t} / \tau_{B}^{T}\right)}+\mathrm{A}_{\mathrm{F}}^{\mathrm{RC}} \times \mathrm{e}^{\left(-\mathrm{t} / \tau_{F}^{R C}\right)} \quad(\text { Eq. 5) }
$$

Regression analysis of the decay curves for different PPN/glycoprotein mixtures leads to the preexponential factors and hence to the amount of free and bound PPN. Thus, at 1:1 drug/protein molar ratio a $45 \%$ of $(R)$-PPN is bound to HAAG.

The photobehavior of $(S)$-NPX in the presence of HSA has been investigated by LFP at $308 \mathrm{~nm}$ in PBS/air. In the absence of protein, a complex transient spectrum is observed, with the main maximum at $440 \mathrm{~nm}\left({ }^{3} \mathrm{NPX}^{*}, 1.8 \mu \mathrm{s}\right)$ and a weaker band in the range 550$650 \mathrm{~nm}\left(\mathrm{NPX}^{+}\right)$. However, in the presence of different amounts of albumin, only ${ }^{3} \mathrm{NPX} *$ is observed, with the kinetic traces at 440 decaying at longer timescales. At 1:1 molar ratio, a satisfactory fitting is obtained considering two monoexponential terms (Eq. 2), whereas at higher drug/protein ratios, three lifetime values are necessary to obtain a good fitting of the decay signal (Eq. 3). This is ascribed to the presence of two long-lived NPX@HSA species, in addition to free NPX. Fitting of the decay curves for NPX/HSA ratios from 1:0.15 to 1:1 allows determining the values of the preexponential coefficients, corresponding to free, site-I bound, and site-II-bound drug. By contrast, a different behavior is observed in the presence of HAAG, where the binding degree is markedly lower. Thus, with equimolar amounts of glycoprotein, two triplet lifetimes are found (Eq. 4), corresponding to free and bound drug; the binding degree is $19 \%$.

Then, NPX, PPN, and CIN have been employed for the assessment of drug distribution in binary protein systems. Upon LFP of the three drugs in the presence or absence of proteins, the naphthalene triplet excited state at $420-440 \mathrm{~nm}$ is always detected, although the decay kinetics is structure- and medium-dependent. Experiments performed 
in HSA/HAAG show that when the two proteins are present simultaneously in the same medium, fitting of the decay traces is clearly consistent with a distribution of the drug between the different biological compartments and the bulk solution, which correlates well with the known protein affinities of each drug.

The results demonstrate that albumins are the major carriers for NPX, while PPN binds preferentially to glycoproteins. An intermediate situation is found for CIN, which presents comparable affinity for both types of proteins. Figure 6 shows the decay kinetics of drugs and the drug distribution at 1/0.5/0.5 drug/HSA/HAAG molar ratio.

A
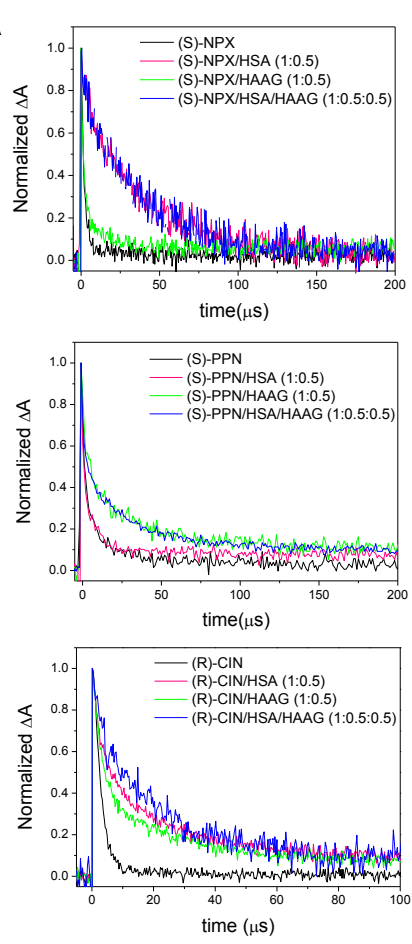

B

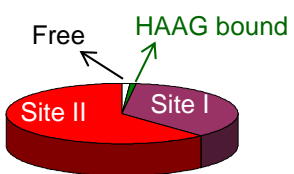

HAAG bound
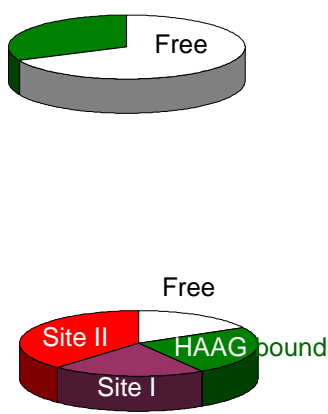

Figure 6. A. Laser flash photolysis of (S)-NPX, (S)-PPN and (R)-CIN ( $\lambda_{\text {exc }}=308 \mathrm{~nm}$ or $266 \mathrm{~nm}, \lambda_{\text {mon }}=420 \mathrm{~nm}$, PBS, air, $A_{308}$ or $266 \mathrm{~nm}=0.3$ ). Kinetic decays in the absence or presence of HSA and/or HAAG. B. Drug distribution obtained after fitting of the decay curves at drug/HSA/HAAG 1:0.5:0.5 molar ratio.

\subsection{Drug-drug interactions revealed by triplet-triplet energy transfer}

The feasibility of drug-drug interactions (DDI) within the binding sites of TP has been probed by TAS, using drug pairs (CIN/PPN and CIN/NAB) complexed within HAAG or BAAG. ${ }^{26}$ The concept involves detection of triplet-triplet energy transfer (TTET), which 
requires a close proximity between the donor and acceptor partners and can only occur within a common biological compartment.

The behavior of CIN/HAAG and PPN/HAAG has been described in the precedent section. Concerning nabumetone (4-[6-methoxy-2-naphthy1]-2-butanone, NAB) it acts as prodrug, being metabolized by the liver to 6-methoxy-2-naphthylacetic acid, the active pharmacological form. ${ }^{27}$ Binding to HAAG and BAAG has also been investigated after LFP at $308 \mathrm{~nm}$ under air; protein bound ${ }^{3} \mathrm{NAB}^{*}$ decays with a $\tau_{\mathrm{T}}$ value of 16.6 in HAAG and $25.8 \mu$ s in BAAG.

In the absence of glycoproteins, the drug mixtures are not soluble in PBS and give rise to turbid suspensions. Complexation of CIN/PPN and CIN/NAB mixtures with glycoproteins is easily followed by naked eye, through a complete solubilization that leads to clear preparations. When a solution of CIN/PPN/HAAG at 1:1:1 molar ratio is submitted to LFP $\left(\lambda_{\text {exc }}=308 \mathrm{~nm}\right.$, air $)$, the decay trace monitored at $450 \mathrm{~nm}$ matches with that of the CIN/HAAG system, with no contribution from ${ }^{3} \mathrm{PPN}^{*}$ in the kinetic decay. A similar situation is observed in the presence of BAAG. Likewise, for the $\mathrm{CIN} / \mathrm{NAB} / \mathrm{glycoprotein}$ systems, the decay traces at $450 \mathrm{~nm}$ are identical to those of the $\mathrm{CIN} /$ glycoprotein mixtures, and ${ }^{3} \mathrm{NAB} *$ does not contribute to the kinetic decay (Figure 7). These results clearly indicate an efficient energy transfer from ${ }^{3} \mathrm{PPN}^{*}$ or ${ }^{3} \mathrm{NAB} *$ to CIN and reveal the occurrence of DDI between drugs that share a common protein binding site. As the TTET process is completed within the duration of the laser pulse $(<$ $20 \mathrm{~ns}$ ), deactivation of the excited triplet states is extremely fast, with a rate constant higher than $5 \times 10^{7} \mathrm{~s}^{-1}$. Such a high value is not compatible with the expectedly slow diffusion inside the protein binding sites and points to formation of pre-associated complexes between donor (PPN or NAB) and acceptor (CIN). This is confirmed by 
theoretical calculations, which combine atomistic and quantum-semiempirical methodologies.
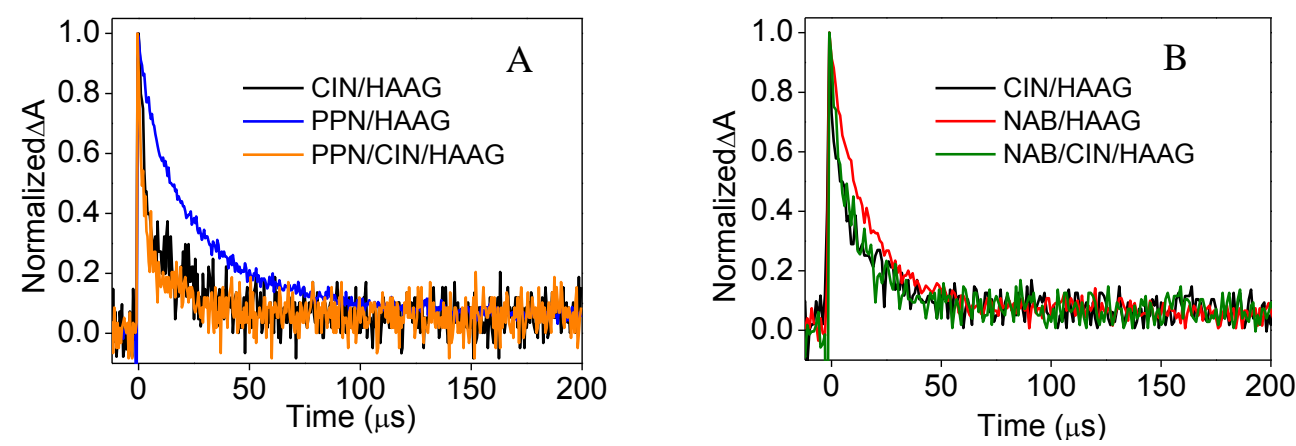

Figure 7. Laser flash photolysis of $\operatorname{drug}_{1} / \mathrm{HAAG}$ and $\operatorname{drug}_{1} / \mathrm{drug}_{2} / \mathrm{HAAG}$ mixtures $\left(\boldsymbol{\lambda}_{\mathrm{exc}}\right.$ $=308 \mathrm{~nm}, \lambda_{\text {mon }}=420 \mathrm{~nm}, \mathrm{PBS}$, air, $\left.\mathrm{A}_{308}=0.3\right)$. A: CIN/PPN and B: CIN/NAB systems.

\section{Reactivity upon protein complexation}

The use of proteins as supramolecular systems to influence the course of organic reactions has attracted increasing interest over the last decades. Specifically, the photoreactivity of encapsulated substrates can be modified by providing protection from attack by oxygen or other external reagents, by imposing conformational restrictions in the binding pockets, or by influencing the stereochemical outcome through the supply of a chiral source. Two examples demonstrating the concept are presented below.

\subsection{Monitoring the enzyme-like activity of HSA}

The $\beta$-1-O-acyl glucuronide metabolites are intrinsically reactive molecules (both in vivo and in vitro) that can undergo a number of reactions; by far, hydrolysis back to the parent aglycon is the predominating process. ${ }^{28}$ This reaction can be catalyzed by several agents ( $\beta$-glucuronidases, esterases, etc.) resulting in a futile cycle, since plasma clearance is the result of competition between conjugation of the parent drug, deconjugation of the glucuronide, and renal clearance. ${ }^{29}$ 
The glucuronidase activity of HSA towards the glucuronides of FBP, $(2 R)$ - and (2S)FBPG (Chart 1) has been investigated by in situ TAS. ${ }^{30}$ It is based on LFP of both glucuronides in the presence of HSA, with the FBP-like TES as reporter. Upon LFP of (2R)-FBPGluc $\left(\lambda_{\text {exc }}=266 \mathrm{~nm}\right.$, PBS, air $)$ a transient spectrum with maximum at $360 \mathrm{~nm}$ is observed, corresponding to TES. Under these conditions, its lifetime is $1.8 \mu$ s. In the presence of different amounts of HSA, LFP of (2R)-FBPG also gives rise to the characteristic $360 \mathrm{~nm}$ signal. Unlike the behavior in the absence of protein, the decays do not follow a first order exponential law, but require biexponential fitting and take place at longer timescales. An important contribution of the $1.8 \mu$ s component is always found, indicating that free $(2 R)$-FBPG is present in the bulk solution within the entire concentration range. The other component, much longer lived $\left(\tau_{\mathrm{T}}=25 \mu \mathrm{s}\right)$, is attributed to $(2 R)-F B P G$ triplet inside the protein cavities. From the $A_{F}$ and $A_{B}$ coefficients, the percentages of the two (free and bound) species with different lifetimes are obtained.

Several observations deserve special comments for their relevant biological implications. First, even at low FBPG/HSA molar ratios, a high amount of glucuronide remains unbound to the protein. This is in good agreement with its enhanced hydrosolubility respect to the parent drug, which facilitates elimination; moreover, no stereodifferentiation takes place in the protein-binding process, since the binding degree determined from $A_{F}$ and $A_{B}$ is very similar for both metabolites. In addition, it is worth mentioning that only a type of microenvironment is provided by the protein for bound FBPG, unlike the case of the parent drug FBP, where two different triplet lifetimes are found corresponding to different binding sites.

After establishing that the methodology is suitable to discriminate between the drug and its metabolite, LFP has been applied to investigate in situ the glucuronidase activity of HSA at physiological temperature $\left(37^{\circ} \mathrm{C}\right)$. Since the amount of complexed glucuronide 
is higher in an excess of albumin, the 1:3.33 ratio is chosen to monitor the reaction. Aliquots of the solution are taken at different times and submitted to LFP. Again, a signal centered at $360 \mathrm{~nm}$ is observed, but now four different $\tau_{\mathrm{T}}$ values are needed to obtain an accurate fitting of the decay: the two values reported above for $(2 R)-\mathrm{FBPG}$ at zero time (1.8 and $25 \mu \mathrm{s})$, plus the two ones ascribed to $(R)$-FBP within the protein binding sites (10.2 and $39.0 \mu \mathrm{s})$. This observation clearly indicates that the hydrolysis process is taking place. No $(R)$-FBP is expected to be present free in solution under these conditions, since inclusion within the protein is known to be complete even at higher molar ratios. The four characteristic triplet lifetimes are now exploited to determine the percentage of compounds in each sample after different reaction times. In the fitting (Eq. 6), $\tau_{\mathrm{F}}{ }^{\mathrm{FBPG}}$ is the triplet lifetime of free $(2 R)-\mathrm{FBPG}, \tau_{\mathrm{I}}^{\mathrm{FBP}}$ and $\tau_{\mathrm{II}}^{\mathrm{FBP}}$ are the corresponding values for $(R)$-FBP within site I and site II, and $\tau_{\mathrm{B}}{ }^{\mathrm{FBPG}}$ is he value for $(2 R)$-FBPG bound to the protein.

$$
\Delta \mathrm{A}=\Delta \mathrm{A}_{0}+\mathrm{A}_{\mathrm{F}} \times \mathrm{e}^{\left(-\mathrm{t} / \tau_{\mathrm{F}}^{\mathrm{FPG}}\right)}+\mathrm{A}_{\mathrm{B}} \times \mathrm{e}^{\left(-\mathrm{t} / \tau_{\mathrm{B}}^{\mathrm{FPG}}\right)}+\mathrm{A}_{\mathrm{I}} \times \mathrm{e}^{\left(-\mathrm{t} / \tau_{\mathrm{I}}^{\mathrm{EPP}}\right)}+\mathrm{A}_{\mathrm{II}} \times \mathrm{e}^{\left(-\mathrm{t} / \tau_{\mathrm{II}}^{\mathrm{EP}}\right)}
$$

Assuming that $\mathrm{A}_{\mathrm{I}} / \mathrm{A}_{\mathrm{II}}$ remains constant for $\mathrm{FPB}$, the preexponential factors and consequently the percentages of $(R)$-FBP and (2R)-FBPGluc, can be obtained. The course of the reaction, expressed as the percentages of the various species at different times, is represented in Figure 8.

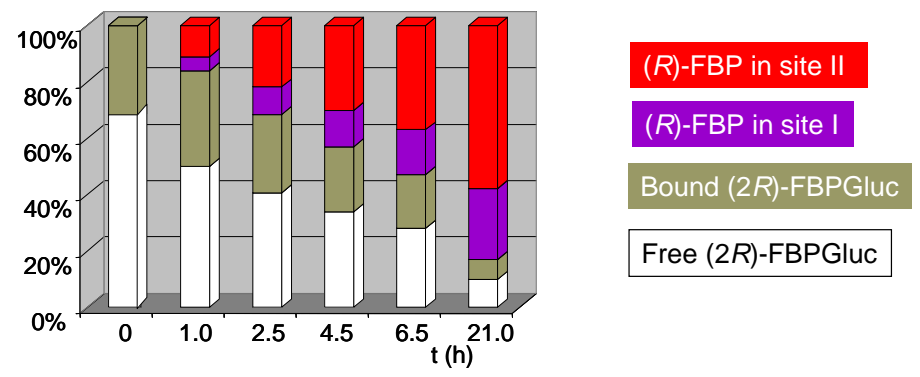

Figure 8. Percentages of free and bound (2R)-FPBG, as well as $(R)$-FBP in site I and site II in the presence of $\mathrm{HSA}$ at $37^{\circ} \mathrm{C}$, obtained using Eq. 6 . 
Similar studies conducted at $22{ }^{\circ} \mathrm{C}$ in the presence of protein or at $37{ }^{\circ} \mathrm{C}$ in the absence of HSA show a much slower process.

\subsection{Enhanced drug photostability of the ligand upon protein encapsulation}

Photooxidation of 9-anthaceneacetic acid (ANA, Chart 1) has been examined using different transport proteins such as HSA, BSA, HAAG and BAAG at 1:1 molar ratio. ${ }^{31}$ Irradiation of ANA in aerated PBS solutions yields anthraquinone through TTET from ${ }^{3}$ ANA* to molecular oxygen, generating singlet oxygen $\left({ }^{1} \mathrm{O}_{2}\right)$. This species reacts with the central anthracene ring of ANA leading to an endoperoxide which undergoes rearrangement followed by cleavage of the 9-substituent to afford anthraquinone (ANQ), as summarized in Scheme 2.

$$
\begin{aligned}
& \mathrm{ANA} \stackrel{\mathrm{hv}}{\longrightarrow}{ }^{1} \mathrm{ANA}^{*} \\
& { }^{1} \mathrm{ANA}^{*} \stackrel{\mathrm{ISC}}{\longrightarrow}{ }^{3} \mathrm{ANA}^{*} \\
& { }^{3} \mathrm{ANA}^{*}+{ }^{3} \mathrm{O}_{2} \rightarrow \mathrm{ANA}+{ }^{1} \mathrm{O}_{2} \\
& \mathrm{ANA}+{ }^{1} \mathrm{O}_{2} \rightarrow \mathrm{ANA}-\mathrm{O}_{2} \rightarrow \text { ANQ }
\end{aligned}
$$

Scheme 2. Generation and reactivity of singlet oxygen following irradiation of ANA.

Within proteins, photooxidation is markedly slower than in bulk solution; the most remarkable effect is found for serum albumins, where the reaction rate constant is one order of magnitude lower. This important protection provided by protein cavities is attributed to a slower diffusion of oxygen inside the protein. In pure PBS, ionic transient species such as the radical anion $\left(\lambda_{\mathrm{abs}}=350\right.$ and $\left.420 \mathrm{~nm}\right)$, the radical cation $\left(\lambda_{\mathrm{abs}}=600\right.$ $750 \mathrm{~nm})$ and hydrated electron $\left(\lambda_{\mathrm{abs}}=700 \mathrm{~nm}\right)$ can be observed (Figure 9A), together 
with the triplet excited state $\left(\lambda_{\max }=420 \mathrm{~nm}\right)$, which is clearly predominating within HSA (Figure 9B).
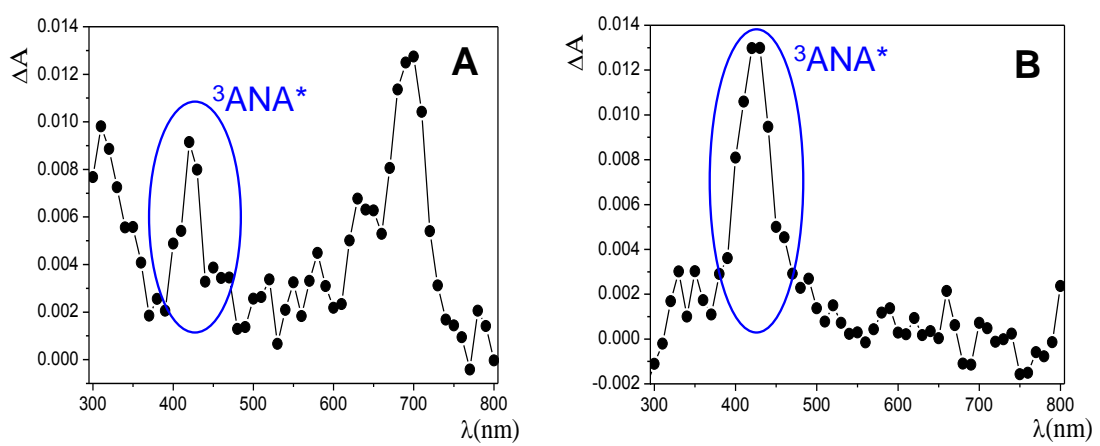

Figure 9. Laser flash photolysis of ANA $\left(\lambda_{\text {exc }}=355 \mathrm{~nm}\right.$, PBS, air, $\left.\mathrm{A}_{355}=0.3\right)$. A: PBS solution B: In the presence of HSA at 1:1 molar ratio.

A dramatic stereodifferentiation in the photooxidation process has been found in the related 9-anthracenepropanoic acids $(S)$ - and $(R)$-ANP (Chart 1). For instance, in the presence of HSA the reaction rate constant is higher for the $(S)$-enantiomer, leading to a kinetic resolution with enhancement of the less reactive $(R)$-ANP. A similar behavior is observed within glycoproteins (Figure 10). From a mechanistic point of view, involvement of singlet oxygen in the photooxidation is confirmed by the remarkable acceleration of reaction rates in $\mathrm{D}_{2} \mathrm{O}$, in agreement with the longer lifetime of ${ }^{1} \mathrm{O}_{2}$ in this medium. Overall, the retarded photooxidation within the protein binding sites is in good agreement with the lower rate constants obtained for quenching of encapsulated ${ }^{3} \mathrm{ANP} *$ by oxygen. Further experiments using selectively excited photosensitizers that are not present in the same microenvironments as ANP demonstrate that photooxidation is compartmentalized. 
- No protein (PBS)

(S)-ANP/HAAG

$(R)-\mathrm{ANP} / \mathrm{HAAG}$

(S)-ANP/BAAG

$\Delta(R)-\mathrm{ANP} / \mathrm{BAAG}$

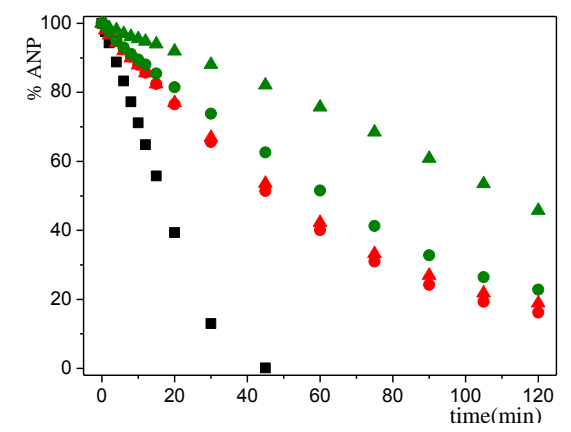

Figure 10. Kinetics of irradiation of ANP in aqueous media/air $\left(\lambda_{\mathrm{exc}}>350 \mathrm{~nm}\right)$ in the presence and in the absence of glycoprotein (1:1 drug/protein molar ratio).

\section{Conclusions}

Binding to proteins plays an important role in the pharmacological activity, biodistribution and metabolism of drugs. Drug/protein supramolecular assemblies can be advantageously investigated by photophysical methods (noteworthy transient absorption spectroscopy) when the drugs of interest contain a photoactive chromophore. This is particularly useful when such chromophore can be selectively excited at long wavelengths, where the aromatic amino acids of the proteins do not absorb. Since triplet excited states exhibit strongly medium-dependent properties, they can be used as reporters to probe the microenvironments defined by the lipophilic protein binding sites, where their formation is strongly favored over generation of ionic species upon laser flash

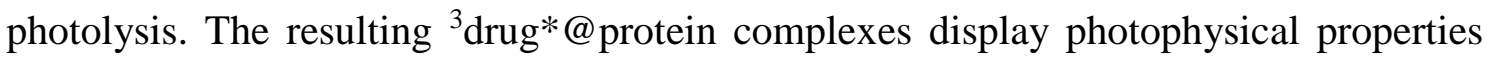
that can be exploited to gather information on conformational restrictions, interaction strength, or protection from attack by oxygen or other agents. Interestingly, the triplet lifetimes of protein-complexed drugs are remarkably long (even under aerobic conditions), which provides a broad dynamic range for identification of distinct populations or for chiral discrimination. The use of proteins as supramolecular vessels modifies the photoreactivity of encapsulated substrates by controlling the exchange flow of reactants between the bulk solution and the protein binding sites, by imposing 
conformational restrictions, by making use of the amino acid residues as active centers, or by supplying a chiral source.

In this review, some examples of the use of TES to obtain relevant information have been described. The technique has also been successfully employed for other purposes, such as the drug photobinding to proteins in photoallergy, ${ }^{32}$ determination of enantiomeric composition in mixtures of enantiomers ${ }^{33}$ or inhibition of ligand deprotonation after protein encapsulation. ${ }^{34}$

\footnotetext{
${ }^{1}$ Scaiano, J. C., Nanosecond Laser Flash Photolysis: a Tool for Physical Organic Chemistry, In: Studies in Reactive Intermediate Chemistry; R. A. Moss, M. S. Platz, M. Jones, Eds.; John Wiley \& Sons, Inc., Hoboken, NJ, USA, 2005.

2 T. Peters, All About Albumins: Biochemistry Genetics and Medical Applications, Academic Press, San Diego, 1995.

${ }^{3}$ Kopecky, V. Jr.; Ettrich, R.; Hofbauerova, K.; Baumruk, V. Structure of Human a1-Acid Glycoprotein and Its High-Affinity Binding Site, Biochem. Biophys. Res. Commun. 2003, 300, 41-46.

${ }^{4}$ Matsumoto, K.; Sukimoto, K.; Nishi, K.; Maruyama, T.; Suenaga, A.; Otagiri, M. Characterization of Ligand Binding Sites on the alpha-1-Acid Glycoprotein in Humans, Bovines and Dogs, Drug. Metab. Pharmacokinet. 2002, 17, 300-306.

${ }^{5}$ Olson, R. E.; Christ, D. D. Plasma Protein Binding of Drugs, Anпи. Rep. Med. Chem. 1996, 31, 327-336.

${ }^{6}$ Otagiri, M. A Molecular Functional Study on the Interactions of Drugs with Plasma Proteins, Drug. Metab. Pharmacokinet. 2005, 20, 309-323.

${ }^{7}$ Fasano, M.; Curry, S.; Terreno, E.; Galliano, M.; Fanali, G.; Narciso, P.; Notari, S.; Ascenzi, P. The Extraordinary Ligand Binding Properties of Human Serum Albumin, Life 2005, 57, 787-796.

${ }^{8}$ Kopecky, V. Jr.; Ettrich, R.; Hofbauerova, K.; Baumruk, V. Structure of Human a1-Acid Glycoprotein and its High-Affinity Binding Site. Biochem. Biophys. Res. Commun. 2003, 300, 41-46.

${ }_{9}^{9}$ Israili, Z. H.; Dayton, P. G. Human alpha-1-Glycoprotein and its Interactions with Drugs, Drug Metab. Rev. 2001, 33, 161-235.

${ }^{10}$ Vuignier, K.; Schappler, J.; Veuthey, J.-L.; Carrupt, P.-A.; Martel, S. Drug-Protein Binding: a Critical Review of Analytical Tools, Anal. Bioanal. Chem. 2010, 398, 53-66.

${ }^{11}$ Cosa, G.; Scaiano, J. C. Laser Techniques in the Study of Drug Photochemistry, Photochem. Photobiol. 2004, 80, 2, 159-174.

${ }^{12}$ Mandula, H., Mohan, J., Parepally, R., Feng, R., Smith, Q.R., Role of Site-Specific Binding to Plasma Albumin in Drug Availability to Brain J. Pharmacol. Exp. Ther. 2006, 317, 667-675.

${ }^{13}$ Zhang, Q., Huang, Y., Zhao, R., Liu, G., Chen, Y. Determining Binding Sites of Drugs on Human Serum Albumin using FIA-QCM, Biosens. Bioelectron. 2008, 24, 48-54.

${ }^{14}$ Flarakos, J., Morand, K.L., Vouros, P., High-Throughput Solution-Based Medicinal Library Screening against Human Serum Albumin, Anal. Chem. 2005. 77, 1345-1343.

${ }^{15}$ Brogden, R.N.; Heel, R.C.; Speight, T.M.; Avery, G.S. Flurbiprofen: a Review of its Pharmacological Properties and Therapeutic Use in Rheumatic Diseases, Drugs 1979, 18, 417-438.

${ }^{16}$ Jiménez, M. C.; Miranda, M. A.; Tormos, R.; Vayá, I. Characterisation of the Lowest Singlet and Triplet Excited States of S-Flurbiprofen, Photochem. Photobiol. Sci. 2004, 3, 1038-1041.

${ }^{17}$ Vayá, I.; Bueno, C. J.; Jiménez, M. C.; Miranda, M. A. Use of Triplet Excited States for the Study of Drug Binding to Human and Bovine Serum Albumins, ChemMedChem 2006, 1, 1015-1020.

${ }^{18}$ Itoh, T.; Saura, Y.; Tsuda, Y.; Yamada H. Stereoselectivity and Enantiomer-Enantiomer Interactions in the Binding of Ibuprofen to Human Serum Albumin, Chirality 1997, 9, 643-649.

${ }^{19}$ Jiménez, M. C.; Miranda, M. C.; Vayá, I. Triplet excited states as chiral reporters for the binding of drugs to transport proteins, J. Am. Chem. Soc. 2005, 127, 10134-10135

${ }^{20}$ Pérez-Ruiz, R.; Bueno, C. J.; Jiménez, M. C.; Miranda, M. A. In situ Transient Absorption Spectroscopy to Assess Competition between Serum Albumin and alpha-1-Acid Glycoprotein for Drug Transport, $J$. Phys. Chem. Lett. 2010, 1, 829-833.
} 
${ }^{21}$ Pérez-Ruiz, R.; Alonso, R.; Nuin, E.; Andreu, I.; Jiménez, M. C.; Miranda, M. A. Naphthalene Triplet Excited State as a Probe for the Assessment of Drug Distribution in Binary Protein Systems, J. Phys. Chem. B 2011, 115, 4460-4468.

22 Torres P. U., Cinacalcet HCl: a Novel Treatment for Secondary Hyperparathyroidism Caused by Chronic Kidney Disease, J. Ren. Nutr. 2006, 16, 253-258.

${ }^{23}$ Duggan K. C., Walters M. J., Musee J., Harp J. M., Kiefer J. R., Oates J. A., Marnett L. J., Molecular Basis for Cyclooxygenase Inhibition by the non-Steroidal anti-Inflammatory Drug Naproxen". J. Biol. Chem. 2010, 285, 34950-34959.

${ }^{24}$ Ananth, J.; Lin, K. M. Propranolol in Psychiatry. Therapeutic Uses and Side Effects, Neuropsychobiology 1986, 15, 20-27.

${ }^{25}$ Nuin, E.; Andreu, I.; Torres, M. J.; Jiménez, M. C.; Miranda, M. A. Enhanced Photosafety of Cinacalcet upon Complexation with Serum Albumin J. Phys. Chem. B 2011, 115, 1158-1164.

${ }^{26}$ Nuin, E.; Jiménez, M. C.; Sastre, G.; Andreu, I.; Miranda, M. A. Drug-Drug Interactions within Protein Cavities Probed by Triplet-Triplet Energy Transfer, J. Phys. Chem. Lett. 2013, 4, 1603-1607.

${ }^{27}$ Martínez, L. J.; Scaiano, J. C. Characterization of the Transient Intermediates Generated from the Photoexcitation of Nabumetone: a Comparison with Naproxen, Photochem. Photobiol. 1998, 68, 646-651.

${ }^{28}$ Bailey, M. J.; Dickinson, R. G. Acyl Glucuronide Reactivity in Perspective: Biological Consequences, Chem. Biol. Interact. 2003, 145, 117-137.

${ }^{29}$ Spahn-Langguth, H.; Benet, L. Z. Acyl Glucuronides Revisited: Is the Glucuronidation Process a Toxification as Well as a Detoxification Mechanism?, Drug. Metab. Rev. 1992, 24, 5-47.

${ }^{30}$ Bueno, C. J.; Jiménez, M. C.; Miranda, M. A. In Situ Transient Spectroscopy for the Study of Glucuronidase Activity within Serum Albumin, J. Phys. Chem. B 2009, 113, 6861-6865.

${ }^{31}$ Alonso, R.; Yamaji, M.; Jiménez, M. C., Miranda, M. A. Enhanced Photostability of the Anthracene Chromophore in Aqueous Medium upon Protein Encapsulation, J. Phys. Chem. B 2010, 114, 11363-11369.

${ }^{32}$ Montanaro, S.; Lihaubet-Vallet, V.; Blanca, M.; Jiménez, M. C.; Miranda, M. A. Photonucleophilic Addition of the $\varepsilon$-Amino Group of Lysine to a Triflusal Metabolite as a Mechanistic Key to Photoallergy Mediated by the Parent Drug, ChemMedChem 2009, 4, 1196-1202.

${ }^{33}$ Vayá, I.; Bueno, C. J., Jiménez, M. C.; Miranda, M. A. Determination of Enantiomeric Compositions by Transient Absorption Spectroscopy using Proteins as Chiral Selectors, Chem. Eur. J. 2008, 14, 1128411287.

${ }^{34}$ Bonancía, P.; Vayá, I.; Jiménez, M. C.; Miranda, M. A. Intraprotein Formation of a Long Wavelength Absorbing Complex and Inhibition of Excited-State Deprotonation in a Chiral Hydroxybiphenyl, J. Phys. Chem. B 2012, 116, 14839-14843. 